\title{
Exploration on teaching reform of theory curriculum for engineering specialties
}

Yan Zhang, Wei-min Shen, Chang-yu Shen, Chen-xia Li, Xu-feng Jing, et al.

Yan Zhang, Wei-min Shen, Chang-yu Shen, Chen-xia Li, Xu-feng Jing, Jun Lou, Yan Shi, Shang-zhong Jin, "Exploration on teaching reform of theory curriculum for engineering specialties," Proc. SPIE 10452, 14th Conference on Education and Training in Optics and Photonics: ETOP 2017, 1045232 (16 August 2017); doi: 10.1117/12.2269899

SDIE Event: 14th Conference on Education and Training in Optics and Photonics, ETOP 2017, 2017, Hangzhou, China 


\title{
Exploration on teaching reform of theory curriculum for engineering specialties
}

\author{
Zhang Yan, Shen Wei-min, Shen Chang-yu, Li Chen-xia, Jing Xu-feng, Lou Jun, \\ Shi Yan, Jin Shang-zhong \\ College of Optical \& Electronic Science and Technology, China Jiliang University, \\ Hangzhou, Zhejiang 310018, China
}

\begin{abstract}
The orientation of talents cultivation for local colleges is to train engineering application-oriented talents, so the exploration and practice on teaching reform of theory curriculum was carried out. We restructured the knowledge units basing on numerical solution problems, and chose the software to build algorithm models for improving the analytical and designed ability. Relying on micro video lessons platform, the teacher-student interaction was expanded from class to outside. Also, we programmed new experimental homework, which was suited for process evaluation. The new teaching mode has achieved good effect, and the students' application ability was significantly improved.
\end{abstract}

Keywords: Application-oriented Talents; Theory Curriculum; Computer Aided Instruction (CAI); Process Evaluation

\section{INTRODUCTION}

A theoretical course, especially a Specialized Fundamental Course, always consists of an integrated theoretical system including many principles, which plays a role in laying good foundation for undergraduate. In the progress of Chinese Higher Education from quantity development to quality development, Classified Undergraduate Talents Cultivation has been the important reform approach. As a local undergraduate college, the talent training objective is engineering application-oriented talents. Concentrated on this goal, we have developed talents training program and reformed curriculum content system a teaching method. Through the reform of professional theory curriculum, we intend to improve students' ability of data processing, case analysis, and solution design for solving engineering practice issues.

Based on the specialties of Optoelectronic Information Science and Engineering and Electronic Science and Technology, we have carried out the exploration and practice on teaching reform of theory curriculum and achieved good effect.

\section{METHODS}

2.1 Reorganize the knowledge system, and restructure the knowledge units basing on numerical solution method.

Theoretical teaching contents are too much and class hours are limited, so the contents need to be simplified. However, the simplification of the theory is not simple to replace the original formulas by

14th Conference on Education and Training in Optics and Photonics: ETOP 2017, edited by Xu Liu,

Xi-Cheng Zhang, Proc. of SPIE Vol. 10452, 1045232 - (c) 2017 ICO, IEEE, OSA, SPIE

CCC code: $0277-786 \mathrm{X} / 17 / \$ 18 \cdot$ doi: $10.1117 / 12.2269899$

Proc. of SPIE Vol. 10452 1045232-1 
the approximate formulas or use linearization method. We should simplify the complicated derivation process and use computer for numerical calculation, even the formulas can be explained by the way of analogy and illustration, which helps students to understand the principles better. From the view point of engineering application, the final requirement is to obtain data, so the accuracy of the data is very important and the advantage of computer data processing is prominent. In order to obtain data conveniently, the knowledge system should be restructured basing on the application orientation and numerical calculation techniques. So we separated contents into many typical units, which construct complete system from simple to complex, and every unit can be solved by numerical method respectively. For example, the imaging process of each sphere is similar for a complex optical system composed of multiple spheres, so we can consider one sphere as a basic unit and use computer to calculate repeatedly. The solution of single spherical imaging is easy, and repeated calculations can be achieved by computer.

\subsection{Improve students' analytical and designed ability basing on Computer Aided Instruction} (CAI).

CAI method can make all the relevant physical characteristics concentrating on one interface, and the relations among these characteristics can be shown as numerical or graphic forms, which are intuitive and easy to understand. One can try to change different parameters to observe the variation of the results, thus determine the influence factors after many tries. We have named it "search method", which can be applied for theoretical analysis. In fact, the engineering data does not need to be very accurate, but is permitted in proper accuracy range. The learning process is simplified using "search method" instead of "analytical method", and all the work including calculation and analysis can be finished by computer. This method is very similar to design, and students must learn to choose proper application software to finish complex calculations. If the multimedia materials such as curves, graphs and animations are created basing on calculations, students even can "first observe then study". In other words, students can first find regularities and phenomena through computer operation, and then clear the principles through teachers' explanation. In order to facilitate students' study, teachers must compile handouts in accordance with the new knowledge units and offer sample documents including numerical algorithms and computer programs for all solvable issues.

\subsection{Expand teacher-student interaction from class to outside relying on micro-video lessons platform.}

Innovation often begins from imitation. However, it is hard for a student to complete the work from understanding abstract formulas to establishing calculation models, next to designing interface and multimedia independently. Sample documents provided by teachers are always characterized as follows. (i) The software we choose is familiar or easy to learn for students. (ii) The software interface is visualized and the relationship among all the parameters is clear. (iii) The variations of curve, graph and other parameters can be displayed synchronously. (iv) Necessary descriptions are included. 
Because one sample document only matches one typical issue, it is impossible to explain all the examples within limited class hours, thus the after-class tutoring is necessary. We have recorded many micro-videos uploading the course website for watching conveniently. The videos are short, always within twenty minutes, and easy to record, so one teacher can finish individually. Course website is not only a good platform for teacher-student interaction, but also is a good way to take advantage of spare time for learning.

\subsection{Program new experimental homework, and improve students' initiative through process evaluation.}

The invariant model of "listen-review-exercise" is tiresome for students, and the new learning model of "inquiry-imitation-design" is worth trying. Students not only can listen and ask in class, but also can expand and push relevant content outside class. We believe that inquiry is important than listen, and imitation is an effective method. Students need to do a new type of work named "experimental homework", which is programmed by teachers and finished by computer. This kind of homework is always related to algorithm, interface, action, and coding, so it is similar to doing experiment. The sample documents are guidance, and doing experimental homework is an innovative training method. In order to prevent mutual plagiarism, teachers have programmed several exercises for each issue, and students were required to do different homework. The new instructional design is especially suitable for process evaluation (also known as formative assessment). The students' scores can be measured by the completion of their experimental homework, and their daily performance in-class, online discussions and traditional homework. All the records should be archived and convenient to check. The reform of process evaluation has played an important role to improve learning initiative and enhance application ability.

\section{PRACTICE AND ACHIEVEMENTS}

The complete scheme of teaching reform is shown as Figure 1. The implementation of the teaching reform was first carried out on the course "Elementary Semiconductor Technology". For example, crystal structure and its symmetry is difficult to understand for students, and traditional teaching method is always with the aid of physical models, but now we can show the graphs and animations using Excel, which are derived from accurate calculations. We instructed students first to draw planar lattice graph, then to build three-dimensional stereogram, and last to design animation demo, step by step. This representation is intuitive and accurate, which makes teaching easier and clearer. It is also effective to be applied in the teaching of lattice vibration, band structure, carrier concentration and conductivity calculation, etc. 


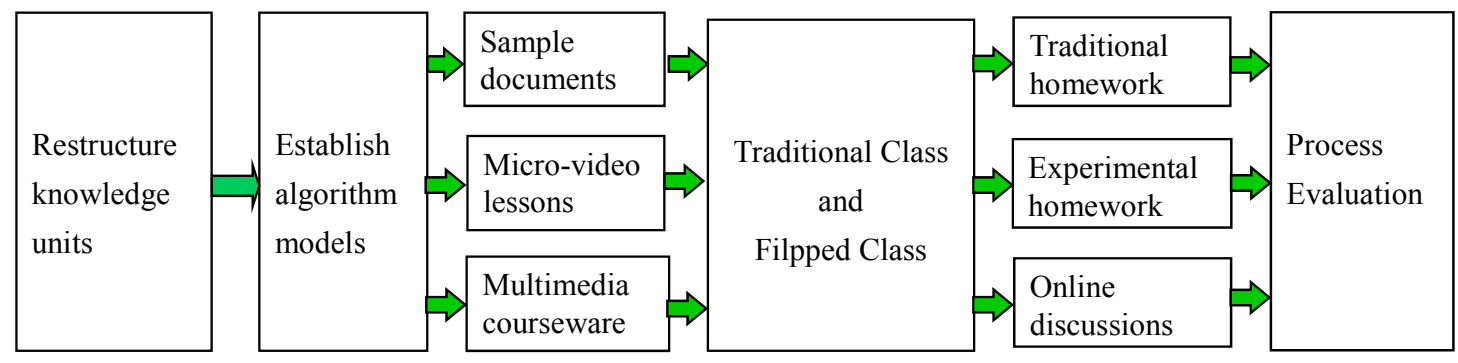

Figure.1 Instructional design of Teaching Reform on theoretical courses

There was no detailed plan for the above course initially, and we gradually summarized and improved methods. However, the teaching reform of theoretical course "Principle of Optics" was performed strictly according the plan, and all the teaching documents were prepared before instruction.

We have carried out questionnaire survey face to all the students. The results show that most students prefer the teaching reform. It reduces the burden to simplify procedure of formula derivation, also the understanding and applications of knowledge can be accomplished by numerical calculation. More than ninety percent students think the sample documents and micro-video lessons are helpful. Moreover, we found that most students' scores of experiment homework were always proportional to the final exam results, which meant that the new teaching method had brought out the enthusiasm of students. Teachers can know the students' learning by the completion of the experimental homework, and it facilitates the tracking and evaluation of teaching process. Furthermore, it is an encouraging thing that students' achievement has been improved than last two terms.

Although the tasks of curriculum reform are heavy, the curriculum resources can be used efficiently once all the teaching documents are prepared. We will carry out teaching reform and practice on more professional theory courses because of the good effects.

\section{ACKNOWLEDGEMENTS}

This work is supported by the Higher Education and Teaching Reformation project of Zhejiang Province under Grant. jg2013071.

\section{REFERENCES}

[1] Hou, A., "The internal logic and mechanism construction of the application oriented undergraduate Colleges," Jiangsu Higher Education, 6, 86-89 (2016).

[2] Zhang, D. L., "Construction of National Quality Course for improving quality," China Higher Education Research, 1, 6-11 (2013).

[3] Xiao, N., "Thoughs on the logic of teaching reform in Chinese Universities," Chine University Teaching, 7, 7-9 (2012).

[4] Zhang, H. T., "Exploration and practice of constructing open practice teaching system for 
engineering applied talents," Chine University Teaching, 8, $73-76$ (2011).

[5] Zou, X. Z., Wang, C. S., Cai, Y. and Zhang, H. E., "Construction of practical teaching system for high quality engineering applied talents," Research and Exploration in Laboratory, 12, 125-127 (2010).

[6] Qu, Z. H., Zhong, X. Q. and Yang, X. H., "Orientation and implementation of talent cultivation in new colleges," China Higher Education Research, 6, 68-69 (2009).

Author: Zhang Yan, Ph. D, Associate Professor, College of Optical \& Electronic Science and Technology, China Jiliang University.

Contact: zyshally@,cjlu.edu.cn +86-13666662620

Address: No. 258, Xueyuan Street, Jianggan District, Hangzhou, Zhejiang 310018, P.R.China 